OPEN ACCESS

Edited by:

Robert Jeenchen Chen,

The Ohio State University,

United States

Reviewed by:

Michael Hofmann,

University of Zurich, Switzerland

Maruti Haranal,

National Heart Institute, Malaysia

*Correspondence: Chang Shu

shuchang@csu.edu.cn

Specialty section:

This article was submitted to Heart Surgery,

a section of the journal

Frontiers in Cardiovascular Medicine

Received: 21 July 2021 Accepted: 06 September 2021 Published: 18 October 2021

Citation:

Wang L, Shu C, Li Q, Li M, He H, LiX, Shi Y, Qiu J, Wang T, Yang C, Wang $M$, Li J, Wang $H$ and Sun $L$ (2021) Application of a Novel Common-lliac-Artery Skirt Technology (CST) in Treating Challenge Aorto-lliac or Isolated lliac Artery Aneurysms.

Front. Cardiovasc. Med. 8:745250. doi: 10.3389/fcvm.2021.745250

\section{Application of a Novel Common-Iliac-Artery Skirt Technology (CST) in Treating Challenge Aorto-lliac or Isolated Iliac Artery Aneurysms}

\author{
Lunchang Wang ${ }^{1,2}$, Chang Shu ${ }^{1,2,3 *}$, Quanming $\mathrm{Li}^{1,2}$, Ming $\mathrm{Li}^{1,2}$, Hao $\mathrm{He}^{1,2}$, Xin $\mathrm{Li}^{1,2}$, \\ Yin Shi ${ }^{4}$, Jian Qiu ${ }^{1,2}$, Tun Wang ${ }^{1,2}$, Chenzi Yang ${ }^{1,2}$, Mo Wang ${ }^{1,2}$, Jiehua Li $^{1,2}$, Hui Wang ${ }^{1,2}$ \\ and Likun Sun ${ }^{1,2}$ \\ ${ }^{1}$ Department of Vascular Surgery, The Second Xiangya Hospital of Central South University, Changsha, China, ${ }^{2}$ Vascular \\ Disease Institute of Central South University, Changsha, China, ${ }^{3}$ Department of Vascular Surgery, Fuwai Hospital, Chinese \\ Academy of Medical Sciences \& Peking Union Medical College, Beijing, China, ${ }^{4}$ Department of Vascular Surgery, Fuwai \\ Yunnan Cardiovascular Hospital, Kunming, China
}

Purpose: To report a novel common-iliac-artery skirt technology (CST) in treating challenge iliac artery aneurysms.

Methods: When required healthy landing zone of common iliac artery $(C I A)$ is not available, CST is a strategy to exclude the internal iliac artery (IIA) and prevent IIA reflux without need of embolization. Patients who received endovascular aneurysm repair (EVAR) in our center from 2014 to 2020 were retrospectively screened, and patients treated with CST or with IIA embolization (IIAE) were enrolled.

Results: After retrospective screen of 524 EVAR patients, 39 CST patients, 26 IIAE patients, and 7 CST + IIAE patients were enrolled in this study. CST group suggested to have more aged, hyperlipemia, and smoking patients than IIAE group. Two groups had comparable maximal diameter of abdominal aorta (AA), CIA, EIA, but larger diameter of IIA (CST $19.82 \pm 2.281$ vs. IIAE $27.82 \pm 3.401, p=0.048$ ), and CIA bifurcation (CST $25.01 \pm 1.316$ vs. IIAE $29.76 \pm 2.775, p=0.087$ ) was found in IIAE group. Anatomy of $79.5 \%$ of CST patients and $92.3 \%$ of IIAE patients ( $p=0.293$ ) was not suitable for potential use of iliac branch device. CST group had significant shorter surgery time (CST $97.42 \pm 3.891$ vs. IIAE $141.0 \pm 8.010, p<0.001$ ), shorter hospital stay (CST 15.35 \pm 0.873 vs. IIAE $19.32 \pm 1.067, p=0.009)$, lower in-hospital [CST 0\% (0/39) vs. IIAE $11.5 \%(3 / 26), p=0.059$ ] and 1-year follow-up stent related MAEs [CST 6.7\% (2/30) vs. IIAE 28.6\% (6/21), $p=0.052$ ], but comparable mortality and stent related MAEs for all-cohort follow-up analysis comparing to IIAE group. In our study, a lower in-hospital buttock claudication (BC) rate for CST (CST 20.5\% vs. IIAE 46.2\%, $p=0.053$ ) and a comparable erectile dysfunction (ED) rate (CST 10.3\% vs. IIAE 23.1\%, $p=0.352$ ) were found between CST and IIAE groups. After 1 year, both groups had about one third relief of BC symptoms [CST 33.3\% (4/12) vs. IIAE 30.7\% (4/13), $p=1.000$ ]. Subgroup analysis of 14 patents concomitant with IIA aneurysm in CST group and the 7 CST + IAE patients 
were carried out, and no difference was found in mortality, stent MAEs, sac dilation, or reintervention rate. Last, illustration of seven typical CST cases was presented.

Conclusion: In selected cases, the CST is a safe, feasible-and-effective choose in treating challenge iliac artery aneurysms and preventing IIA endoleak.

Keywords: aorto-iliac artery aneurysm, iliac artery aneurysm, internal iliac artery, novel technology, endovascular, aortic surgery

\section{INTRODUCTION}

Endovascular aneurysm repair (EVAR) has become the preferred way to treat abdominal aortic aneurysms (AAA) with suitable anatomy and life expectancy $(1,2)$. Iliac artery aneurysms (IAAs) are commonly coexisting with AAAs as aorto-iliac aneurysms in about $10-30 \%$ of AAA $(3,4)$, which poses significant clinical and technical challenges during $\operatorname{EVAR}(5,6)$. Specifically, if the distal common iliac artery (CIA) does not present an adequate healthy landing zone, exclusion of internal iliac artery (IIA) or hypogastric artery is often required. Though benefits of preserving IIAs are now well-acknowledged and different strategies, devices, such as iliac branch device (IBD), are developed (7). Nevertheless, such techniques would significantly increase the surgery time and complexity and could be limited by anatomical constraints, technique experience, and availability of grafts. Remarkably, only $40.9 \%$ patients were suitable for use of iliac branch devices in an on-label fashion according to the manufacturer's instructions (8), and IBDs are not available in China until March 2021. And also, aneurysmal patients often complicated with cardiovascular diseases that require a feasibleand-effective procedure, particular for ruptured AAA patients $(9,10)$.

On the other hand, exclusion of the IIA is widely used and has been proven safe (11-15), and minimal adverse events of possible ischemic complications can be achieved as long as the contralateral IIA is fluent $(14,15)$. Coil or plug embolization of IIA, direct extension of graft to EIA, or combination of both is the main method for IIA exclusion. Extension of graft to EIA has risk of type II endoleak (16), while coil embolization is challenging and time-consuming with unfeasible anatomy. Here, we described a novel common-iliac-artery skirt technique (CST) in treating challenge aorto-iliac or isolated iliac artery aneurysms, which preserve the advantages of extension of graft to EIA and diminish risk of type II endoleak.

\section{MATERIALS AND METHODS}

\section{Enrollment and Data Collection}

We retrospectively screened patients diagnosed with abdominal or iliac artery aneurysm and treated by endovascular procedures from 2014 to 2020 in our center. Among them, patients treated with CST or IIA embolization (IIAE) were enrolled in this study. Demographic data, risk factors and comorbidity, diagnosis, preprocedure, procedure and in-hospital data, and follow-up results were analyzed. All patients received informed consent before the surgery, and this study was approved by the Institutional Review Board and the Medical Ethics Committee of our hospital.

A pre-surgical computed tomography angiogram (CTA) was used in all patients to assess extent of aneurysmal disease, tortuosity of the iliac vessels, and patency of the internal and external iliac arteries. Diameter of abdominal aortic and ipsilateral iliac arteries of IIAE or CST were measured from outer-wall to outer-wall of the long-axis. Length of CIA was retrospective collected by DSA with help of centimeter sizing pigtail catheter (Cook Medical Inc). Diameter and length were measured by two authors, and the average value of two authors' was used. Evaluation of erectile dysfunction was only carried out on male patients who are under 70 s and had normal erectile function before surgery. Surgery time was calculated from anesthesia induction to the last frame of angiography. Two DSA rooms were routinly used by our group and due to the deficiency of one old-fashion DSA system; unfortunately, not all patients had radiation time and dosage data, so they were not analyzed in this study.

\section{Indications for CST or IIAE}

CIAA without adequate healthy landing zone was the indication for CST or IIAE. Surgical method selection was based on general condition, comorbilities and vascular anatomy of each patient, and also patient's own will. In the IIAE group, embolization was performed preferentially via a contralateral approach before EVAR. IIAE was achieved using embolization coils (Cook Medical Inc) at the proximal site of the bifurcation of primary branches.

\section{Primary and Secondary End Points}

Technical successful rate was defined as primary end points, and technique success including complete exclusion of aneurysms and no endoleak from IIA during surgery.

Secondary end points include surgery time, hospital stay, mortality, stent related major adverse events (MAEs), and reintervetion, while stent related MAEs include endoleak, limb occlusion, stent migration, contralateral IIA occlusion, and pelvic ischemia.

\section{Follow-Up and Statistics}

Survival and reintervention events were followed by telephone calls directly with patients regularly. CTA imaging was carried out with available follow-up patients. Data are presented as median (range) or mean \pm standard deviation. Data between two groups were analyzed using the Student $t$ test, Chi-square, or Fisher's exact test analysis. A two-tailed $P$ value of $<0.05$ indicated statistical significance. 


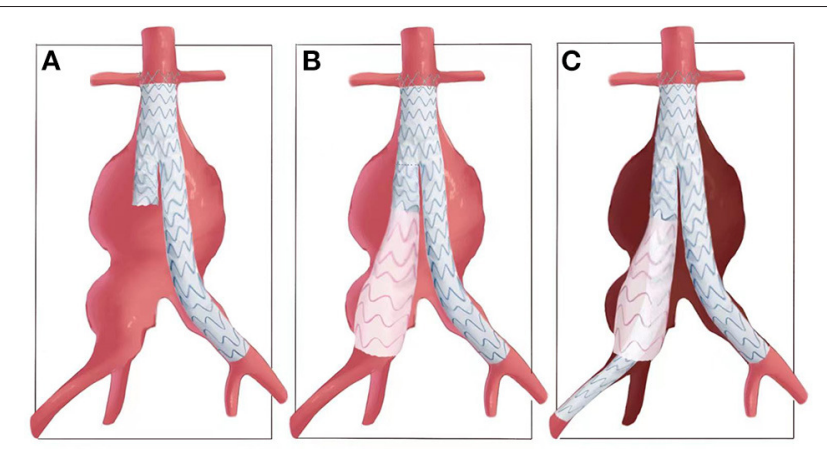

FIGURE 1 | Illustration of common-iliac-artery skirt technology. (A) An aorto-iliac artery aneurysm and release of the bifurcated main body graft. (B) Then, a flared iliac limb is deployed at aneurysmal CIA and with its distal end locating at and sealing the iliac bifurcation, which is named as skirt-limb. (C) Another iliac limb with the same proximal diameter of skirt limb is deployed overlap with skirt-limb and elongated to EIA, with IIA excluded and possible reflux of IIA was restricted between the two iliac limbs.

\section{RESULTS}

\section{Procedure of a Classical}

Common-Iliac-Artery Skirt Technique (CST)

As illustrated in Figure 1, after deployment of the aortic bifurcated grafts, with the shorter leg placed in aneurysmal CIA side, a flared iliac extension limb (skirt-limb) was deployed at aneurysmal CIA and with its distal end locating at and sealing the iliac bifurcation. The proximal end of skirt-limb with an identical diameter of the bifurcated shorter leg was placed within the leg, the diameter of distal end of skirt-limb should not smaller than the iliac bifurcation, and a $10 \%$ oversize of diameter was recommended but not obligatory.

And then, another iliac limb graft with the same proximal diameter of skirt limb was deployed overlap with skirt-limb and elongated to EIA, with IIA excluded and possible reflux of IIA restricted between two iliac limbs (Figure 1). Relationship of the two iliac stent grafts was similar with lady's skirt and leg, respectively.

For challenge iliac aneurysm, aortic extension configuration or cuff, or double CST skill can also be used in CST, which will be shown hereinafter.

\section{Demographics and Pre-Procedural Data}

Retrospective screen found 524 patients received EVAR in our center from 2014 to 2020, and 39 CST patients, 26 IIAE patients, and 7 CST + IIAE patients were enrolled in this study. Given the small sample size of CST + IIAE group, regular statistical analysis only carried out between CST and IIAE group and most data of CST + IIAE group was not presented in the text, which but can be provided upon reasonable inquiry.

Demographic data, risk factors and comorbidity analysis suggested that CST group trend to have more aged, hyperlipidemia, and smoking patients than IIAE group, though not all data were statistically significant (Table 1). Male patients were overwhelming in both group, $97.4 \%$ for CST and $96.2 \%$ for

TABLE 1 | Demographics and preprocedural data.

\begin{tabular}{|c|c|c|c|}
\hline & CST & IIAE & $p$ \\
\hline Male & $97.4 \%(38 / 39)$ & $96.2 \%(25 / 26)$ & 1.000 \\
\hline Age & $70.23 \pm 0.969$ & $67.54 \pm 1.383$ & 0.105 \\
\hline Hypertension & $66.7 \%(26 / 39)$ & $65.4 \%(17 / 26)$ & 1.000 \\
\hline Hyperlipidemia & $46.2 \%(18 / 39)$ & $23.1 \%(6 / 26)$ & 0.071 \\
\hline Smoking & $94.8 \%(33 / 36)$ & $61.5 \%(16 / 26)$ & 0.002 \\
\hline CAD & $20.5 \%(8 / 39)$ & $23.1 \%(6 / 26)$ & 1.000 \\
\hline COPD & $23.1 \%(9 / 39)$ & $11.5 \%(3 / 26)$ & 0.334 \\
\hline CKD & $2.56 \%(1 / 39)$ & $0 \%(0 / 26)$ & 1.000 \\
\hline History of $\mathrm{Cl}$ & $15.4 \%(6 / 39)$ & $3.85 \%(1 / 26)$ & 0.228 \\
\hline Diagnosis & & & 0.253 \\
\hline $\mathrm{AAA}+\mathrm{CIAA}$ & $30.8 \%$ (12/39) & 26.9\% (7/26) & I \\
\hline$A A A+C I A A+I I A A$ & $35.8 \%(14 / 39)$ & $19.2 \%(5 / 26)$ & I \\
\hline CIAA & $15.4 \%(6 / 39)$ & $15.4 \%(4 / 26)$ & I \\
\hline $\mathrm{CIAA}+\mathrm{IIAA}$ & $18 \%(7 / 39)$ & $38.5 \%(10 / 26)$ & I \\
\hline \multicolumn{4}{|l|}{ Diameter of (mm) } \\
\hline AAA & $40.25 \pm 2.218$ & $34.40 \pm 3.203$ & 0.129 \\
\hline ClA & $41.22 \pm 7.600$ & $35.59 \pm 2.893$ & 0.585 \\
\hline CIA length & $39.45 \pm 3.676$ & $33.79 \pm 3.492$ & 0.271 \\
\hline $\mathrm{ClA}_{\mathrm{b}}$ & $25.01 \pm 1.316$ & $29.76 \pm 2.775$ & 0.087 \\
\hline$\| \mathrm{A}$ & $19.82 \pm 2.281$ & $27.82 \pm 3.401$ & 0.048 \\
\hline EIA & $9.951 \pm 0.174$ & $10.34 \pm 0.290$ & 0.231 \\
\hline Exclusion of IBD & $79.5 \%$ (31/39) & $92.3 \%(24 / 26)$ & 0.293 \\
\hline
\end{tabular}

$C A D$, coronary artery disease; $C K D$, chronic kidney disease; Cl, cerebral infarction; IBD, iliac branch device.

IIAE group. Average age was $70.23 \pm 0.969$ for CST vs. $67.54 \pm$ 1.383 for IIAE group $(p=0.105)$. Hypertension percentage was $66.7 \%$ for CST vs. $65.4 \%$ for IIAE $(p=1.000)$. CST group had a trend to have more patients with hyperlipidemia (CST 46.2\% vs. IIAE $23.1 \%, p=0.07$ ), but two groups had no difference on coronary artery disease percentage (CST $20.5 \%$ vs. IIAE $23.1 \%$, $p=1.000)$. CST group had a significant higher proportion of smoking patients (CST $94.8 \%$ vs. IIAE $61.5 \%, p=0.002$ ) and non-significant higher proportion of COPD patients (CST 23.1\% vs. IIAE $11.5 \%, p=0.334$ ). Both groups had low prevalence of chronic kidney disease, while CST group had more patients with history of cerebral infarction (CST $15.4 \%$ vs. IIAE $3.85 \%, p=$ 0.228), though not significant.

Comparison of diagnosis and vascular anatomy confirmed that all enrolled patients were diagnosed with AAA concomitant with IAA or isolated IAA. Patients were divided into four categories based on diagnosis: AAA + CIAA, AAA + CIAA + IIAA, CIAA, CIAA + IIAA. No significant difference was found on proportion of diagnosis between CST and IIAE group ( $p$ $=0.253$ ).

Two groups had comparable maximal diameter of AA (CST $40.25 \pm 2.218$ vs. IIAE $34.40 \pm 3.203, p=0.129$ ), CIA (CST $41.22 \pm 7.600$ vs. IIAE $35.59 \pm 2.893, p=0.585$ ), EIA (CST $9.95 \pm 0.174$ vs. IIAE $10.34 \pm 0.290, p=0.231$ ) (Table 1), but larger diameter of IIA (CST $19.82 \pm 2.281$ vs. IIAE $27.82 \pm 3.401$, $p=0.048$ ), and CIA bifurcation (CST $25.01 \pm 1.316$ vs. IIAE $29.76 \pm 2.775, p=0.087$ ) was found in IIAE group. Based on 
TABLE 2 | Procedure and in-hospital data.

\begin{tabular}{|c|c|c|c|}
\hline & CST & IIAE & $p$ \\
\hline \multicolumn{4}{|l|}{ Primary end points } \\
\hline Technique successful rates & $100 \%$ & $100 \%$ & 1.000 \\
\hline \multicolumn{4}{|l|}{ Secondary end points } \\
\hline \multicolumn{4}{|l|}{ In hospital results } \\
\hline Surgery time (min) & $97.42 \pm 3.891$ & $141.0 \pm 8.010$ & $<0.001$ \\
\hline Hospital stay (day) & $15.35 \pm 0.873$ & $19.32 \pm 1.067$ & 0.009 \\
\hline ICU stay rate & $5.13 \%(2 / 39)$ & 7.69\% (2/26) & 1.000 \\
\hline Distal diameter of Skirt stent (mm) & $23.90 \pm 0.344$ & / & / \\
\hline 20 & $12.8 \%(5 / 39)$ & / & / \\
\hline 24 & $74.4 \%(29 / 39)$ & / & / \\
\hline 28 & $12.8 \%(5 / 39)$ & / & / \\
\hline 28-28 cuff & $7.69 \%(3 / 39)$ & l & / \\
\hline Save of contralateral IIA & $92.3 \%(36 / 39)$ & $76.9 \%(20 / 26)$ & 0.140 \\
\hline All-cause mortality & $0 \%(0 / 39)$ & $0 \%(0 / 26)$ & 1.000 \\
\hline Stent related MAEs & $0 \%(0 / 39)$ & $11.5 \%(3 / 26)$ & 0.059 \\
\hline Endoleak & $0 \%(0 / 39)$ & $0 \%(0 / 26)$ & 1.000 \\
\hline Limb occlusion & $0 \%(0 / 39)$ & $3.8 \%(1 / 26)$ & 0.400 \\
\hline Migration & $0 \%(0 / 39)$ & $0 \%(0 / 26)$ & 1.000 \\
\hline Contralateral IIA occlusion & $0 \%(0 / 39)$ & $3.8 \%(1 / 26)$ & 0.400 \\
\hline Ineffective IIAE & $0 \%(0 / 39)$ & $3.8 \%(1 / 26)$ & 0.400 \\
\hline Reintervetion & $0 \%(0 / 39)$ & $3.8 \%(1 / 26)$ & 0.400 \\
\hline Buttock claudication & $20.5 \%(8 / 39)$ & $46.2 \%(12 / 26)$ & 0.053 \\
\hline Erectile dysfunction & $10.3 \%(3 / 29)$ & $23.1 \%(3 / 13)$ & 0.352 \\
\hline Colonic ischemia & $0 \%(0 / 39)$ & $0 \%(0 / 26)$ & 1.000 \\
\hline Pelvic necrosis & $0 \%(0 / 39)$ & $0 \%(0 / 26)$ & 1.000 \\
\hline
\end{tabular}

the inclusion/exclusion criteria of the Gore Excluder Iliac Branch Endoprosthesis Trial and the Cook Zenith Iliac Branch Device Clinical Study $(17,18)$, enrolled patients in our study had a high exclusion rate for potential IBD use (CST 79.5\% vs. IIAE 92.3\%, $p=0.293)$.

\section{Procedure and In-Hospital Data}

All surgeries were elective and performed under general anesthesia and by the same surgery team. Access was routinely achieved by cut-down femoral artery exposure. For primary end points, both groups had $100 \%$ technique successful rates and complete exclusion of aneurysms, though one case of IIAE group only achieved partial IIA embolization, but no endoleak from IIA was found after angiography (Table 2). For secondary end points, CST group had significant shorter surgery time (CST $97.42 \pm$ 3.891 vs. IIAE $141.0 \pm 8.010, p<0.001$ ) and shorter hospital stay (CST $15.35 \pm 0.8734$ vs. IIAE $19.32 \pm 1.067, p=0.009$ ) compared to IIAE group. Both groups had low rate of Intensive Care Unit stay [CST 5.13\% (2/39) vs. IIAE 7.69\% (2/26), $p=$ $1.000]$. By analyzing skirt-limb's distal diameter, we found that $74.4 \%$ had a diameter of $24 \mathrm{~mm}$, and $12.8 \%$ had a diameter of 28 , including three $28-28 \mathrm{~mm}$ cuff. Preserve of contralateral IIA was guaranteed as long as suitable anatomy existed, and both groups had high rate of saving contralateral IIA (CST 92.3\% vs. IIAE $76.9 \%, p=0.140)$.
TABLE 3 | Follow-up results.

\begin{tabular}{lccc}
\hline & CST & IIAE & p \\
\hline 1-year results & & & \\
Follow-up rates & $82.1 \%(32 / 39)$ & $92.3 \%(24 / 26)$ & 0.296 \\
All-cause mortality & $6.3 \%(2 / 32)$ & $12.5 \%(3 / 24)$ & 0.639 \\
Stent related MAEs & $6.7 \%(2 / 30)$ & $28.6 \%(6 / 21)$ & 0.052 \\
$\quad$ Endoleak & $0 \%(0 / 30)$ & $0 \%(0 / 21)$ & 1.000 \\
$\quad$ Limb occlusion & $3.3 \%(1 / 30)$ & $14.3 \%(3 / 21)$ & 0.293 \\
$\quad$ Migration & $0 \%(0 / 30)$ & $0 \%(0 / 21)$ & 1.000 \\
$\quad$ Contral IIA occlusion & $3.3 \%(1 / 30)$ & $14.3 \%(3 / 21)$ & 0.293 \\
$\quad$ Endotension or Aneurysm sac dilation & $0 \%(0 / 30)$ & $0 \%(0 / 21)$ & 1.000 \\
Reintervetion & $3.3 \%(1 / 30)$ & $4.8 \%(1 / 21)$ & 1.000 \\
Relived buttock claudication & $37.5 \%(3 / 8)$ & $33.3 \%(4 / 12)$ & 1.000 \\
Relived erectile dysfunction & $0 \%(0 / 3)$ & $33.3 \%(1 / 3)$ & $/$ \\
Colonic ischaemia & $0 \%(0 / 30)$ & $0 \%(0 / 21)$ & 1.000 \\
Pelvic necrosis & $0 \%(0 / 30)$ & $0 \%(0 / 21)$ & 1.000 \\
All-corhot results & & & \\
Follow-up (month) & $13-82 \mathrm{~m}$ & $12-79 \mathrm{~m}$ & 0.203 \\
Median follow-up & 35 & 26 & 0.203 \\
All-cause mortality & $9.38 \%(3 / 32)$ & $12.5 \%(3 / 24)$ & 0.679 \\
Stent related MAEs & $18.8 \%(6 / 32)$ & $25 \%(6 / 24)$ & 0.744 \\
Endoleak & $3.13 \%(1 / 32)$ & $0 \%(0 / 24)$ & 1.000 \\
Limb occlusion & $6.25 \%(2 / 32)$ & $12.5 \%(3 / 24)$ & 0.642 \\
Migration & $3.13 \%(1 / 32)$ & $0 \%(0 / 24)$ & 1.000 \\
Other IIA thrombosis & $6.25 \%(2 / 32)$ & $12.5 \%(3 / 24)$ & 0.642 \\
Endotension or aneurysm sac dilation & $3.13 \%(1 / 32)$ & $0 \%(0 / 24)$ & 1.000 \\
Reintervetion & $6.9 \%(2 / 32)$ & $4.2 \%(1 / 24)$ & 1.000 \\
\hline & & & \\
\hline
\end{tabular}

Analysis of in-hospital results found none mortality for both groups. CST had lower stent related major adverse events compared to IIAE group [CST $0 \%(0 / 39)$ vs. IIAE $11.5 \%$ (3/26), $p=0.059]$. IIAE group had one limb occlusion, one unintended contralateral IIA occlusion, and one ineffective embolization of IIA. No endoleak or stent migration was found in both group. One reintervetion was applied in IIAE group due to limb occlusion. Both groups had pelvic ischemia complications, while CST had lower rate of buttock claudication (BC) (CST 20.5\% vs. IIAE 46.2\%, $p=0.053$ ). Comparable erectile dysfunction (ED) (CST $10.3 \%$ vs. IIAE $23.1 \%, p=0.352$ ) was found, but no severe $\mathrm{BC}$ or $\mathrm{ED}$ require reintervention existed. No colonic ischemia and pelvic necrosis happened (Table 2).

\section{Follow-Up Results}

CST group had $82.1 \%(32 / 39)$ and IIAE group had $92.3 \%(24 / 26)$ $(p=0.296) 1$-year follow-up (Table 3). One-year all causemortality rate was $6.3 \%$ (2/32) for CST vs. $12.5 \%$ (3/24) for IIAE, $p=0.639$. Stent MAEs increased in both groups after 1 year and IIAE group suggested having higher MAE events [CST $6.7 \%$ $(2 / 30)$ vs. IIAE $28.6 \%(6 / 21), p=0.052]$, which is consistent with in-hospital MAEs. In IIAE group, three limb occlusion and three contralateral IIA occlusion happened. In CST group, one limb occlusion and one contralateral IIA occlusion happened. No endoleak, stent migration, endotension or aneurysm sac 
TABLE 4 | Results of different IIAA treatment strategies in CST group.

\begin{tabular}{lccc}
\hline & CST & CST + IIAE & $\boldsymbol{p}$ \\
\hline Diagnosis & & & 0.642 \\
AAA + CIAA + IIAA & $50 \%(7 / 14)$ & $28.6 \%(2 / 7)$ & $/$ \\
CIAA + IIA & $50 \%(7 / 14)$ & $71.4 \%(5 / 7)$ & $/$ \\
Diameter of (mm) & & & \\
CIA & $34.98 \pm 4.175$ & $46.14 \pm 6.244$ & 0.146 \\
CIA & & & \\
IIA & $29.01 \pm 4.179$ & $42.40 \pm 6.047$ & 0.082 \\
IIA > 35 mm & $35.31 \pm 3.823$ & $48.47 \pm 3.230$ & 0.038 \\
Surgery time (min) & $42.9 \%(6 / 14)$ & $100 \%(7 / 7)$ & 0.018 \\
Save of IIA of other side & $100.3 \pm 5.781$ & $120.6 \pm 6.962$ & 0.047 \\
Loss of follow-up & $85.7 \%(12 / 14)$ & $42.9 \%(3 / 7)$ & 0.120 \\
Follow-up (month) & $21.4 \%(3 / 14)$ & $14.3 \%(1 / 7)$ & 1.000 \\
Median FU & $18-70 \mathrm{~m}$ & $15-30 \mathrm{~m}$ & 0.296 \\
All-cause mortality & 25.5 & 22.5 & 0.296 \\
Stent related MAEs & $9.1 \%(1 / 11)$ & $16.7 \%(1 / 6)$ & 1.000 \\
$\quad$ Endoleak & $9.1 \%(1 / 11)$ & $33.3 \%(2 / 6)$ & 0.546 \\
Limb occlusion & $0 \%(0 / 11)$ & $16.7 \%(1 / 6)$ & 1.000 \\
$\quad$ Migration & $0 \%(0 / 11)$ & $16.7 \%(1 / 6)$ & 0.353 \\
Endotension or Aneurysm sac dilation & $0 \%(0 / 11)$ & $16.7 \%(1 / 6)$ & 0.353 \\
Reintervetion & $9.1 \%(1 / 11)$ & $16.7 \%(1 / 6)$ & 1.000 \\
\hline & & &
\end{tabular}

dilation happened for both groups. Both groups only had one reintervetion [CST 3.3\% (1/30) vs. IIAE 4.8\% (1/21), $p=1.000$ ] for symptomatic MAEs. After 1 year, both groups had about one third relief of BC symptoms [CST 33.3\% (4/12) vs. IIAE $30.7 \%(4 / 13), p=1.000]$, and IIAE had $33.3 \%(1 / 3)$ relief of erectile dysfunction.

Analysis of all-cohort follow-up data was also carried out. The median follow-up month for CST was 35 (range 13-82), and 26 (range 12-79) for IIAE group $(p=0.203)$. No difference in mortality (CST $9.38 \%$ vs. IIAE $12.5 \%, p=0.679$ ), stent MAEs (CST $18.8 \%$ vs. IIAE $25 \%, p=0.744$ ), and reintervetion rate (CST $6.9 \%$ vs. IIAE $4.2 \%, p=1.000$ ) was found between two groups. CST group had one type II endoleak and sac enlargement of $5 \mathrm{~mm}$ from inferior mesenteric artery at 2-year follow-up, and regular follow-up was in process according to patient's willing (Table 3).

\section{Results of Different IIAA Treatment Strategies in CST Group}

For patients diagnosed with IIAA, concerns may remain on risk of future IIAA enlargement by only covering the orifice of IIA. So subgroup analysis of 14 patents concomitant with IIA dilation in CST group without IIAE and the 7 CST+IIAE patients were performed (Table 4). The proportion of diagnosis was comparable. CST+IIAE group had larger diameter of CIA bifurcation (CST $29.01 \pm 4.179$ vs. CST+IIAE $42.40 \pm 6.047, p$ $=0.082)$ and IIA (CST $35.31 \pm 3.823$ vs. CST+IIAE $48.47 \pm$ $3.230, p=0.038)$ and higher percentage of IIA $>35 \mathrm{~mm}$ [CST $42.9 \%(6 / 14)$ vs. CST+IIAE 100\% (7/7), $p=0.018]$, and longer surgery time (CST $100.3 \pm 5.781$ vs. CST+IIAE $120.6 \pm 6.962$, $p=0.047)$. But all-cohort analysis with a median follow-up of 25.5 months for CST and 22.5 months for CST $+\operatorname{IIAE}(p=0.296)$ showed no difference in mortality, stent MAEs, sac dilation, or reintervetion between two groups. Notably, both groups had one sac enlargement event. The one in CST group was described previously, and the sac enlargement in IIAAE group was thought to result from endotension, and open surgery was performed after 5-year follow-up (Table 4).

\section{Illustration of Typical and Challenge Cases With CST}

To illustrate the application and advantage of CST, we presented several typical and challenge CST cases. Cases 1-3 showed three cases of AAA concomitant with a huge or short CIAA (Figure 2), and cases 1 and 3 were concomitant with IIAA at the same time. A Medtronic 16-28 mm flared skirt-limb placed in CIA and a 16-13 mm iliac limb extended to EIA was used to seal the CIAA and exclusion of one side IIA. From the DSA, we could notice that the reflux of IIA was contained between the skirt graft and the extended iliac limb (white arrow in Figures $\mathbf{2 C}, \mathbf{H}, \mathbf{M}$ ). One year follow-up results all showed no endoleak or aneurysm sac dilation, and thrombosis formed in space between skirt-limb and extended iliac limb (white arrow in Figures 2E,J,O).

Cases 4 and 5 showed application of CST for isolated CIAAs (Figure 3). Case 4 showed a satisfactory result of sealing a $50.3 \mathrm{~mm}$ CIAA sequentially by a $16-24 \mathrm{~mm}$ graft, a $28-28 \mathrm{~mm}$ iliac limb, and two $16-16 \mathrm{~mm}$ iliac limbs, with the first 16-24 skirt limb located at the orifice of CIA and second 28-28 skirt limb located at the distal bifurcation of CIA. The double skirttechnology fully prevented any potential endoleaks to the aortic sac. Case 5 showed a long and huge CIA with $61 \mathrm{~mm}$ maximal diameter, which was also excluded by double CST skill, composed by a graft series of a 16-24, a 16-16, another 16-24 and 16-16 mm graft.

Cases 6 and 7 showed different strategies in treating IIAA in CST group (Figure 4). Both cases showed bilateral CIAA concomitant with IIAAs. For case 6, a series of 28-28, 16-13, 16-13 mm grafts were formed a CST for the right side, and a 1624 followed with a $16-13 \mathrm{~mm}$ graft were used for the left side. Post-surgery and 1-year follow-up results showed complete seal of aneurysm, exclusion of IIA blood flow, no endoleak, and no aneurysm sac enlargement. For case 7 , both CIAAs were treated by a $28-28$ graft combined with a $16-13 \mathrm{~mm}$ iliac limb after coil embolization of the IIA. One-year follow-up showed satisfactory seal of aneurysm and complete embolization of IIA, though light type II endoleak from inferior mesenteric artery existed.

\section{DISCUSSION}

Strategies of treating IIA changed during the development of AAA treatment. At the early stage of AAA open surgery, preservation of at least one IIA was thought to be necessary and bypass of IIA to graft was used both in our center and others (19). Then, with the development of endovasular surgery as first line therapy and more and more data of safety on IIA exclusion, save of IIA was secondary to providing adequate landing zone and complete sealing of aneurysms $(11,13,15)$. However, with development of new devices and technologies, preservation of 


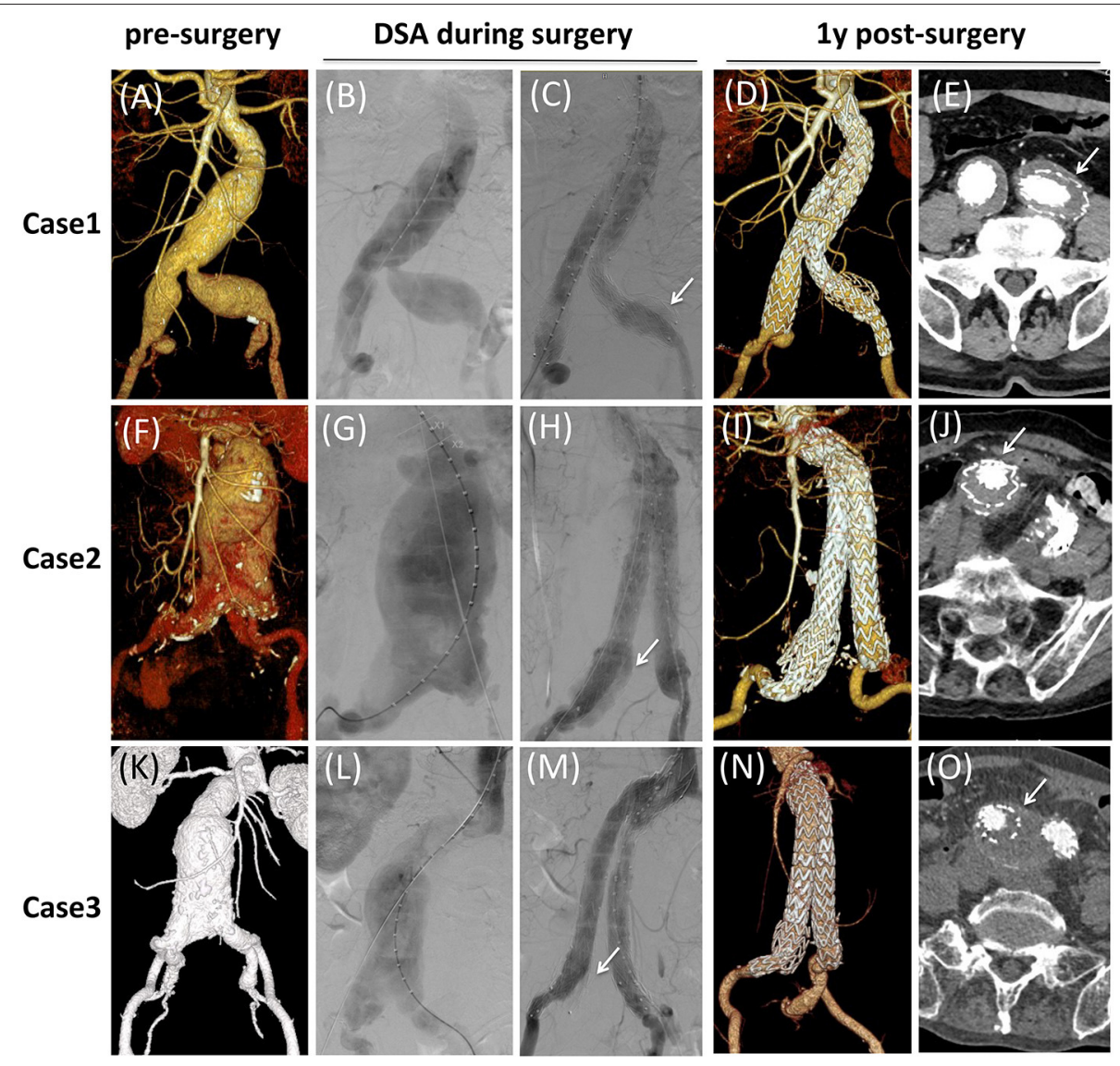

FIGURE 2 | Typical cases of CST for aorto-iliac aneurysms. (A,F,K) Pre-surgical CTA 3D reconstruction. (B,G,L) DSA of aneurysms. (C,H,M) DSA after CST procedure. The white arrow indicates reflux of IIA was contained between the skirt graft and the extended iliac limb. (D,I,N) One-year follow-up CTA 3D reconstruction. (E,J,O) Transverse section of 1-year follow-up CTA. The white arrow indicates thrombosis formed in space between skirt graft and extended iliac limb.

IIA and effort to decrease $\mathrm{BC}$ and $\mathrm{ED}$ become more and more important. With development of IBDs and improved Quality Control of AAA surgery, a preservation era of IIA is thought coming (20).

But, such new techniques still could be limited by anatomical constraints, technique experience, and availability of grafts. For example, IBDs were not available in China until March 2021. After full evaluation of patient condition, activity level, length, tortuosity and thrombus burden of iliac arteries, and cost, exclusion of IIA was still an indispensable technology in selected cases. And here we reported a novel common iliac artery skirt technology for feasible-and-effective IIA exclusion.

Based on retrospective analysis of data from 39 CST and 26 IIAE patients, our experience showed both groups had comparable primary end points and CST had better secondary end points, including significant short surgery time and hospital stay, lower in-hospital and 1-year stent MAEs, comparable mortality, and stent MAEs for all-cohort follow-up. Results of CST showed clear advantages over IIAE in excluding IIA and at the same time preventing the occurrence of type II endoleaks. Actually, the use of a flared grafts in excluding IIA with or without embolization also published in case reports, either with an upside-down or reversed bell-bottom technique (21-24). But, either a manually unsheathed-and-mounted procedure or a contralateral femoral access and crossover process is needed, respectively, which are not necessary for our CST.

Advantages of CST come from its simplicity and which results in short-time surgery and anesthesia, and less endovascular procedures. Ischemia of pelvic is the main concern and complication for exclusion of hypogastric artery. Literature reports of $\mathrm{BC}$ rate were $36.5 \%$ for bilateral IIA interruption and $27.2 \%$ for unilateral IIA interruption (25), and the erectile dysfunction rate was $12.7 \%$. In our study, the in-hospital BC rate was $20.5 \%$ for CST vs. $46.2 \%$ for IIAE, $p=0.053$ and the ED rate was $10.3 \%$ for CST vs. $23.1 \%$ for IIAE, $p=$ 0.352 . The direct coverage of orifice of IIA was suggested to have lower rate of BC than embolization of IIA in our study, and evidence was also reported in published data $(11,26-$ 28). Nevertheless, the high preserve rate of contralateral IIA in CST should also not be ignored, thought not statistically significant. After 1 year, about one third BC was relived in both groups (CST 37.5\% vs. IIAE 33.3\%, $p=1.000$ ), and which all happened on patients who had a preserved contralateral IAA. Thus, the save of at least one IIA should be guaranteed 


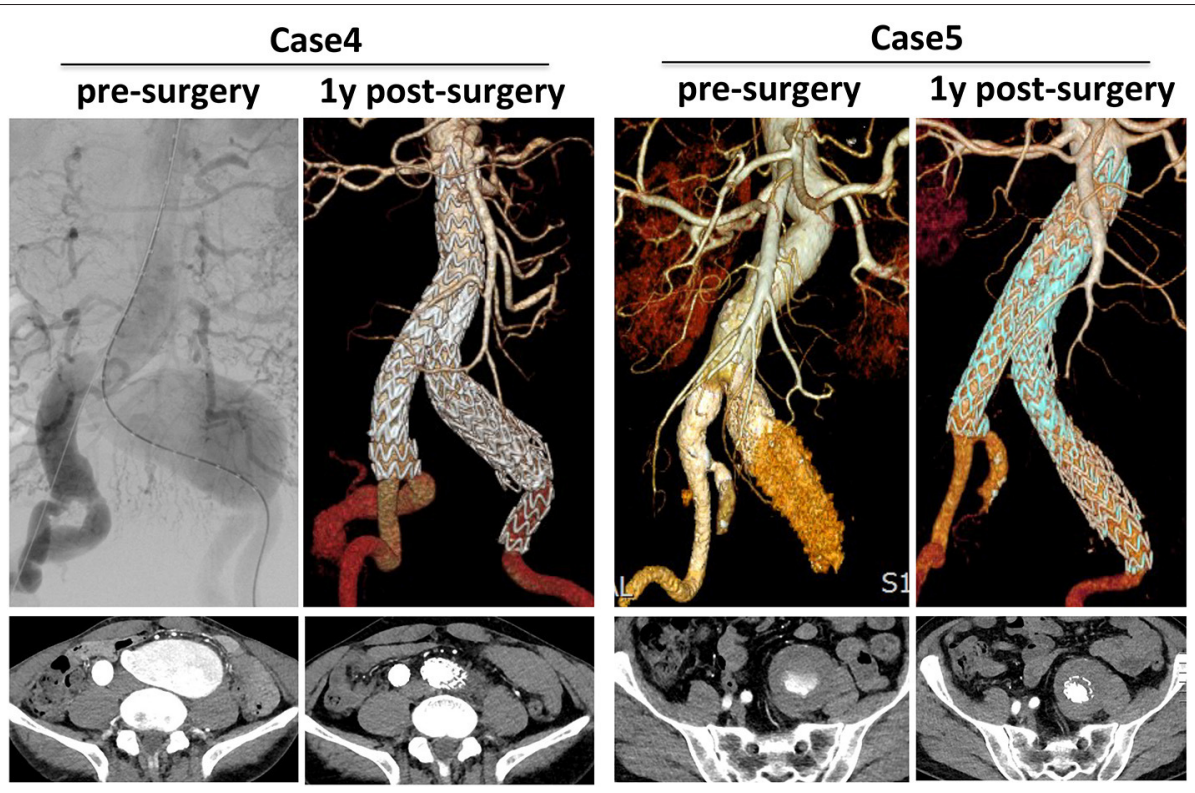

FIGURE 3 | Typical cases of CST for isolated iliac aneurysms. 3D CTA pre-surgery and 1-year post-surgery was compared and corresponding transverse section was also presented.
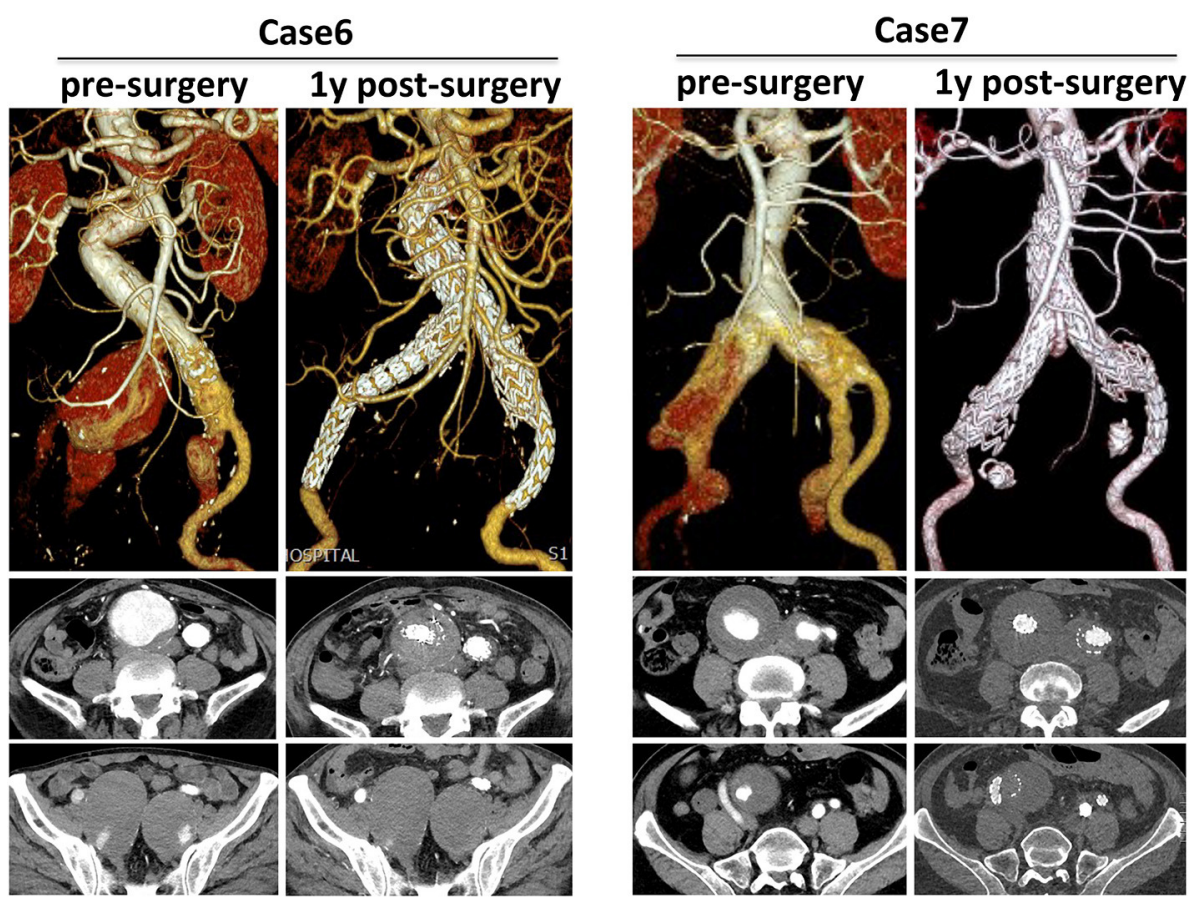

FIGURE 4 | Different strategies in treating IIAA in CST group. 3D CTA pre-surgery and 1-year post-surgery was compared and corresponding transverse section was also presented.

as long as possible, as per guidelines $(1,29)$. And as both CST and IIAE have high and comparable technique successful rate and exclusion of IIA in this study by our group, no difference of mortality and stent MAEs in long term follow-up was found.
Whereas, the critical size for isolated IIAAs treatment is considered to be 3-4 cm (30-32), whether its benefit to treat smaller IIAAs in patients undergoing EVAR for aorta-common iliac aneurysm to avoid the need for secondary interventions is still not clear. And also, for CST group, concerns may 
remain on risk of future IIAA enlargement by only covering the orifice of IAA. In our study, by subgroup analysis of 14 patents concomitant with IIA dilation in CST group without IIAE and the 7 CST+IIAE patients, no difference was found in mortality, stent MAEs, sac dilation, or reintervetion between two groups. However, considering the challenging of secondary interventions for IIAAs after CST, we recommend that CST plus IIA embolization or other IIAAs procedures should be applied for IIAAs $>35 \mathrm{~mm}$ (33).

Overall, the common-iliac-artery skirt technology is simple, since it does not require neither particular endovascular expertise of the operator nor peculiar devices, which has decisive advantage in ruptured patients as a fast and life-saving treatment option. Besides, as the application of CST only restricted by the diameter of CIA bifurcation and the $10 \%$ oversize was not strictly needed for the skirt limb, CST was available for majority cases by using available flared iliac limbs and cuffs (maximal $36 \mathrm{~mm}$ ). Comparing to IIAE, our experience showed CST had advantages in reducing surgery time, hospital stay, stent related MAEs, and $\mathrm{BC}$ rates. Satisfying safety and sealing of CIAAs without type II endoleak was also obtained.

Unilateral application of CST plus a patency or preserved contralateral IIA could be a satisfactory solution for most challenge iliac artery aneurysms. Nevertheless, larger sample size and long-term follow-up is warranted to assess the durability of the technology and absence of re-interventions.

As a retrospectively study, limitations of this study may include potential selection bias of patients for surgical method. However, comparable results of majority risk factors were found between groups.

\section{CONCLUSION}

In selected cases, the CST can be used for the complete exclusion of challenge iliac artery aneurysms and preventing type II

\section{REFERENCES}

1. Wanhainen A, Verzini F, Van Herzeele I, Allaire E, Bown M, Cohnert T, et al. Editor's choice - European Society for Vascular Surgery (ESVS) 2019 clinical practice guidelines on the management of abdominal aorto-iliac artery aneurysms. Eur J Vasc Endovasc Surg. (2019) 57:893. doi: 10.1016/j.ejvs.2018.09.020

2. Chaikof EL, Dalman RL, Eskandari MK, Jackson BM, Lee WA, Mansour MA, et al. The society for vascular surgery practice guidelines on the care of patients with an abdominal aortic aneurysm. J Vasc Surg. (2018) 67:2-77 e2. doi: 10.1016/j.jvs.2017.10.044

3. Krupski WC, Selzman CH, Floridia R, Strecker PK, Nehler MR, Whitehill TA. Contemporary management of isolated iliac aneurysms. J Vasc Surg. (1998) 28:1-11; discussion-3. doi: 10.1016/S0741-5214(98)70194-6

4. Armon MP, Wenham PW, Whitaker SC, Gregson RH, Hopkinson BR. Common iliac artery aneurysms in patients with abdominal aortic aneurysms. Eur J Vasc Endovasc Surg. (1998) 15:2557. doi: 10.1016/S1078-5884(98)80186-X

5. Hobo R, Sybrandy JE, Harris PL, Buth J, Collaborators E. Endovascular repair of abdominal aortic aneurysms with concomitant common iliac artery aneurysm: outcome analysis of the EUROSTAR Experience. J Endovasc Ther. (2008) 15:12-22. doi: 10.1583/07-2217.1 endoleak from the IIA, especially for those who has advanced age, or contralateral health IIA, or cannot tolerate long-time surgery and is limited in activities, or when preservation of IIA does more harm than good or is not practical.

\section{DATA AVAILABILITY STATEMENT}

The raw data supporting the conclusions of this article will be made available by the authors, without undue reservation.

\section{ETHICS STATEMENT}

The studies involving human participants were reviewed and approved by Ethics Committee of the 2nd Xiangya Hospital, Central South University. The patients/participants provided their written informed consent to participate in this study. Written informed consent was obtained from the individual(s) for the publication of any potentially identifiable images or data included in this article.

\section{AUTHOR CONTRIBUTIONS}

CS, LW, QL, ML, HH, and XL contributed to conception and design of the study. LW, YS, JQ, TW, CY, MW, and JL collected data and organized the database. HW and LS performed the statistical analysis. LW wrote the first draft of the manuscript. All authors contributed to manuscript revision, read, and approved the submitted version.

\section{FUNDING}

This study was supported with National Natural Science Foundation of China for LW (81900423), CS (81870345), and JL (81800400) and Natural Science Foundation of Hunan Province for LW (2020JJ5836) and JL (2019JJ50851).
6. Bannazadeh M, Jenkins C, Forsyth A, Kramer J, Aggarwal A, Somerset $\mathrm{AE}$, et al. Outcomes for concomitant common iliac artery aneurysms after endovascular abdominal aortic aneurysm repair. J Vasc Surg. (2017) 66:13907. doi: 10.1016/j.jvs.2017.02.058

7. Robalo C, Sousa J, Mansilha A. Internal iliac artery preservation strategies in the endovascular treatment of aortoiliac aneurysms. Int Angiol. (2018) 37:346-55. doi: 10.23736/S0392-9590.18.04004-X

8. Gray D, Shahverdyan R, Jakobs C, Brunkwall J, Gawenda M. Endovascular aneurysm repair of aortoiliac aneurysms with an iliac side-branched stent graft: studying the morphological applicability of the Cook device. Eur J Vasc Endovasc Surg. (2015) 49:283-8. doi: 10.1016/j.ejvs.2014.12.021

9. Brewster DC, Retana A, Waltman AC, Darling RC. Angiography in the management of aneurysms of the abdominal aorta. Its value and safety. N Engl J Med. (1975) 292:822-5. doi: 10.1056/NEJM1975041729 21602

10. Olin JW, Melia M, Young JR, Graor RA, Risius B. Prevalence of atherosclerotic renal artery stenosis in patients with atherosclerosis elsewhere. Am J Med. (1990) 88:46N-51N.

11. Bosanquet DC, Wilcox C, Whitehurst L, Cox A, Williams IM, Twine CP, et al. Systematic review and meta-analysis of the effect of internal iliac artery exclusion for patients undergoing EVAR. Eur J Vasc Endovasc Surg. (2017) 53:534-48. doi: 10.1016/j.ejvs.2017.01.009 
12. Lee WA, O'Dorisio J, Wolf YG, Hill BB, Fogarty TJ, Zarins CK. Outcome after unilateral hypogastric artery occlusion during endovascular aneurysm repair. J Vasc Surg. (2001) 33:921-6. doi: 10.1067/mva.2001.114999

13. Lee $\mathrm{C}$, Dougherty $\mathrm{M}$, Calligaro K. Concomitant unilateral internal iliac artery embolization and endovascular infrarenal aortic aneurysm repair. J Vasc Surg. (2006) 43:903-7. doi: 10.1016/j.jvs.2005.12.063

14. Schoder M, Zaunbauer L, Holzenbein T, Fleischmann D, Cejna M, Kretschmer $\mathrm{G}$, et al. Internal iliac artery embolization before endovascular repair of abdominal aortic aneurysms: frequency, efficacy, and clinical results. AJR Am J Roentgenol. (2001) 177:599-605. doi: 10.2214/ajr.177.3.1770599

15. Cynamon J, Lerer D, Veith FJ, Taragin BH, Wahl SI, Lautin JL, et al. Hypogastric artery coil embolization prior to endoluminal repair of aneurysms and fistulas: buttock claudication, a recognized but possibly preventable complication. J Vasc Interv Radiol. (2000) 11:573-7. doi: 10.1016/S1051-0443(07)61608-X

16. Lo RC, Buck DB, Herrmann J, Hamdan AD, Wyers M, Patel VI, et al. Risk factors and consequences of persistent type II endoleaks. J Vasc Surg. (2016) 63:895-901. doi: 10.1016/j.jvs.2015.10.088

17. Pearce BJ, Varu VN, Glocker R, Novak Z, Jordan WD, Lee JT. Anatomic suitability of aortoiliac aneurysms for next generation branched systems. Ann Vasc Surg. (2015) 29:69-75. doi: 10.1016/j.avsg.2014.08.003

18. Karthikesalingam A, Hinchliffe RJ, Malkawi AH, Holt PJ, Loftus IM, Thompson MM. Morphological suitability of patients with aortoiliac aneurysms for endovascular preservation of the internal iliac artery using commercially available iliac branch graft devices. J Endovasc Ther. (2010) 17:163-71. doi: 10.1583/09-2942.1

19. Unno N, Inuzuka K, Yamamoto N, Sagara D, Suzuki M, Konno H. Preservation of pelvic circulation with hypogastric artery bypass in endovascular repair of abdominal aortic aneurysm with bilateral iliac artery aneurysms. J Vasc Surg. (2006) 44:1170-5. doi: 10.1016/j.jvs.2006.08.011

20. Kouvelos G, Nana P, Spanos K. Commentary: time to preserve the iliac arteries during EVAR. J Endovasc Ther. (2020) 27:8267. doi: $10.1177 / 1526602820940784$

21. Leon LR Jr, Mills JL Sr. Successful endovascular exclusion of a common iliac artery aneurysm: off-label use of a reversed Cook Zenith extension limb stentgraft. Vasc Endovasc Surg. (2009) 43:76-82. doi: 10.1177/1538574408322661

22. van der Steenhoven TJ, Heyligers JM, Tielliu IF, Zeebregts CJ. The upside down gore excluder contralateral leg without extracorporeal predeployment for aortic or iliac aneurysm exclusion. J Vasc Surg. (2011) 53:173841. doi: 10.1016/j.jvs.2010.11.108

23. Koike Y, Nishimura J, Hase S, Yamasaki M. The upside down endurant iliac limb stent graft for treatment of a common iliac artery aneurysm. Vasc Endovasc Surg. (2014) 48:58-60. doi: 10.1177/1538574413510615

24. Mazzaccaro D, Righini P, Zuccon G, Modafferi A, Malacrida G, Nano G. The reversed bell-bottom technique (ReBel-B) for the endovascular treatment of iliac artery aneurysms. Catheter Cardiovasc Interv. (2020) 96:E47983. doi: $10.1002 / \mathrm{ccd} .29140$

25. Kouvelos GN, Katsargyris A, Antoniou GA, Oikonomou K, Verhoeven EL. Outcome after interruption or preservation of internal iliac artery flow during endovascular repair of abdominal aorto-iliac aneurysms. Eur J Vasc Endovasc Surg. (2016) 52:621-34. doi: 10.1016/j.ejvs.2016.07.081

26. Chitragari G, Schlosser FJ, Ochoa Chaar CI, Sumpio BE. Consequences of hypogastric artery ligation, embolization, or coverage. J Vasc Surg. (2015) 62:1340-7 e1. doi: 10.1016/j.jvs.2015.08.053

27. Farahmand P, Becquemin JP, Desgranges P, Allaire E, Marzelle J, RoudotThoraval F. Is hypogastric artery embolization during endovascular aortoiliac aneurysm repair (EVAR) innocuous and useful? Eur J Vasc Endovasc Surg. (2008) 35:429-35. doi: 10.1016/j.ejvs.2007.12.001

28. Jean-Baptiste E, Brizzi S, Bartoli MA, Sadaghianloo N, Baque J, Magnan PE, et al. Pelvic ischemia and quality of life scores after interventional occlusion of the hypogastric artery in patients undergoing endovascular aortic aneurysm repair. J Vasc Surg. (2014) 60:40-9, 9 e1. doi: 10.1016/j.jvs.2014.01.039

29. Chaikof EL, Brewster DC, Dalman RL, Makaroun MS, Illig KA, Sicard GA, et al. The care of patients with an abdominal aortic aneurysm: the Society for Vascular Surgery practice guidelines. J Vasc Surg. (2009) 50:S249. doi: 10.1016/j.jvs.2009.07.001

30. Dix FP, Titi M, Al-Khaffaf $H$. The isolated internal iliac artery aneurysm-a review. Eur J Vasc Endovasc Surg. (2005) 30:119-29. doi: 10.1016/j.ejvs.2005.04.035

31. Rana MA, Kalra M, Oderich GS, de Grandis E, Gloviczki P, Duncan AA, et al. Outcomes of open and endovascular repair for ruptured and nonruptured internal iliac artery aneurysms. J Vasc Surg. (2014) 59:63444. doi: $10.1016 /$ j.jvs.2013.09.060

32. Laine MT, Bjorck M, Beiles CB, Szeberin Z, Thomson I, Altreuther M, et al. Few internal iliac artery aneurysms rupture under $4 \mathrm{~cm}$. J Vasc Surg. (2017) 65:76-81. doi: 10.1016/j.jvs.2016.06.109

33. Kliewer M, Plimon M, Taher F, Walter C, Hirsch K, Falkensammer J, et al. Endovascular treatment of hypogastric artery aneurysms. J Vasc Surg. (2019) 70:1107-14. doi: 10.1016/j.jvs.2018.12.048

Conflict of Interest: The authors declare that the research was conducted in the absence of any commercial or financial relationships that could be construed as a potential conflict of interest.

Publisher's Note: All claims expressed in this article are solely those of the authors and do not necessarily represent those of their affiliated organizations, or those of the publisher, the editors and the reviewers. Any product that may be evaluated in this article, or claim that may be made by its manufacturer, is not guaranteed or endorsed by the publisher.

Copyright (C) 2021 Wang, Shu, Li, Li, He, Li, Shi, Qiu, Wang, Yang, Wang, Li, Wang and Sun. This is an open-access article distributed under the terms of the Creative Commons Attribution License (CC BY). The use, distribution or reproduction in other forums is permitted, provided the original author(s) and the copyright owner(s) are credited and that the original publication in this journal is cited, in accordance with accepted academic practice. No use, distribution or reproduction is permitted which does not comply with these terms. 